\title{
Photonic-based laser driven electron beam deflection and focusing structures
}

\author{
T. Plettner, ${ }^{*}$ R. L. Byer, and C. McGuinness \\ E.L. Ginzton Laboratories, Stanford University, Stanford, California 94305, USA \\ P. Hommelhoff \\ $M P Q$, Garching, Germany \\ (Received 22 February 2009; published 26 October 2009)
}

\begin{abstract}
We propose a dielectric photonic structure for ultrafast deflection and focusing of relativistic charged particle beams. The structure is designed to transform a free-space laser beam into a deflection force that acts on the free particles with the same optical phase over a distance of travel that is much greater than the laser wavelength. The proposed structure has a two-dimensional geometry and is compatible with existing nanofabrication methods. Deflection fields of $\mathrm{GV} / \mathrm{m}$ magnitude and subfemtosecond switching speeds are expected to be possible from these dielectric structures. With these elements a submeter scale extreme ultraviolet synchrotron source seems feasible.
\end{abstract}

DOI: 10.1103/PhysRevSTAB.12.101302

PACS numbers: 41.75.Jv, 41.75.Ht, 42.25.Bs

\section{INTRODUCTION}

In a prior publication we described a laser-driven dielectric-material deflection microstructure that acts as a building block for a compact undulator [1]. This article explores further aspects of this structure, including its ability for beam focusing, for ultrafast beam streaking, and for beam switching. Generation of beams consisting of electron pulses with sub-fsec duration from laser-driven particle accelerators was observed recently by indirect means [2]. A direct temporal diagnostic is desirable for future studies of laser-driven particle accelerators and for the successful development of other devices that manipulate sub-fsec electron beams. The compactness of the proposed deflection device is possible from the ability of dielectric materials to support ultrashort laser pulses with electric field values in the $\mathrm{GV} / \mathrm{m}$ range without damage. The deflection structure shares a similar geometry with dielectric grating based laser-driven particle accelerators [3] and, hence, can be integrated with these into the same substrate and by the same nanofabrication process.

The proposed deflection structure features three key aspects; first, the generation of a phase-synchronous deflection force that allows for an interaction length that extends far beyond a single wavelength of the laser beam. The synchronicity condition is imposed by a periodic evanescent field. Second, the deflection structure provides a symmetric force pattern that minimizes the electron beam degradation. Finally, the structure is nonresonant, which, as discussed in Sec. III, allows the for application of few-cycle laser pulses.

The dielectric double grating whose cross section is depicted in Fig. 1 supports these properties. Binary quartz

\footnotetext{
*tomas.plettner@gmail.com
}

based gratings have become commercial components [46]. The incoming laser beam travels in the $y$ direction as indicated by the solid arrow in Fig. 1, and its phase front is parallel to the electron beam. To maintain extended overlap with the electron beam along the vacuum channel, the laser beam has to be pulse-front tilted. The grooves of the transmission grating create phase-synchronous diffraction orders inside the vacuum channel. In Sec. II it is shown that these are evanescent. For simplicity only one laser beam is shown, but as discussed in Sec. III, the desired field sym-

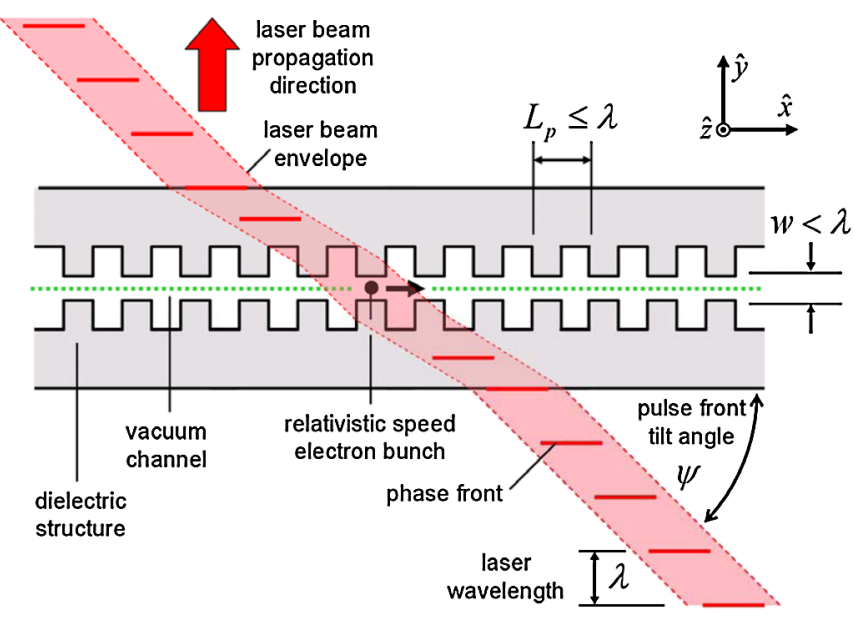

FIG. 1. (Color) Top view of a proposed periodic-phase modulation accelerator structure. The grating grooves are parallel to the $\hat{z}$ axis and the laser beam is traveling parallel to the $\hat{y}$ axis. The electron beam is traveling in the vacuum channel parallel to the $\hat{x}$ axis. The pulse-front tilt causes the laser pulse envelope in the vacuum channel to remain overlapped with the relativistic electron bunch. The substrate is a dielectric material transparent to the laser wavelength in question. 
metry is generated by a pair of laser beams approaching the structure from opposite sides.

Metallic open grating accelerator structures have been studied by Palmer [7], Pickup [8], and other authors. Recently, metallic grating Smith-Purcell undulators for $\mathrm{THz}$ radiation were explored by Lin et al. [9]. The acceleration and synchronicity concepts developed for metallic gratings are applicable to the proposed dielectric doublegrating structure, but one fundamental property that appears with transparent gratings is that the laser can be coupled from within the medium. This allows for a double-grating geometry that has a confined vacuum channel and that, when illuminated from both sides, can provide a field pattern that is symmetric with respect to the electron beam orbit. In addition, the confined vacuum space brings about a different set of boundary conditions for the evanescent field when compared to the traditional, semiopen Smith-Purcell accelerator geometry.

\section{PHASE AND ENVELOPE SYNCHRONICITY CONDITIONS}

We apply a plane-wave field decomposition method to find the diffraction modes that provide a significant deflection and have a phase velocity that is matched to that of the electron beam. The electron beam is assumed to have a velocity $|\vec{v}|=\beta c$, where $\beta$ is smaller than unity. Similar to Palmer's open grating accelerators [7], and as shown in Fig. 2, we allow the grating grooves to have an oblique orientation with respect to the electron beam that is quantified by the angle $\alpha$. The unprimed coordinate system in Fig. 2 is aligned with the structure grooves while the primed is aligned with the electron beam. It is found that $\alpha \neq 0$ is critical for the generation of a nonzero phasesynchronous deflection force (i.e. perpendicular to the nominal beam direction). The geometry is assumed to have infinite extent along the $z$ coordinate. The validity

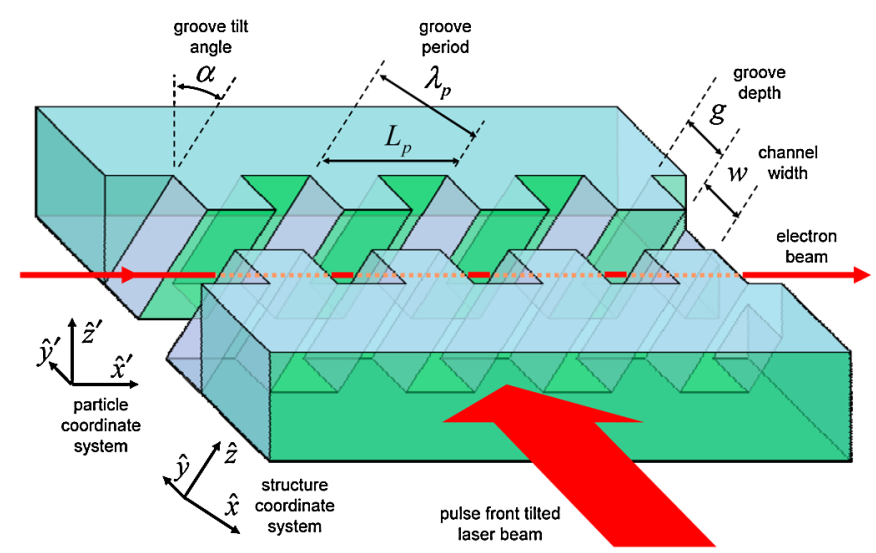

FIG. 2. (Color) Perspective view of the laser-driven dielectric deflection structure. The oblique orientation of the grating grooves with respect to the electron beam is a key aspect of the proposed structure. of this approximation is presented in the Appendix. The electromagnetic fields can then be approximated by independent transverse electric (TE) and transverse magnetic (TM) polarizations. Here the TE polarization corresponds to the mode with the electric field parallel to the grating grooves. The period of the grating, denoted by $\lambda_{p}$, is $L_{p}=$ $\lambda_{p} / \cos \alpha$.

First assume that the laser beam is a TM-polarized monochromatic plane wave of angular frequency $\omega$. Let this wave impinge on the grating structure with an angle $\varphi$ as shown in Fig. 3 (note that $\varphi$ is not the pulse-front tilt angle $\psi$ as shown in Fig. 1). The electric field of such an incident wave is described by

$$
\vec{E}(x, y, z, t)=\hat{P} E_{0} e^{i(\omega t-k x \sin \varphi-k y \cos \varphi)-i \phi} .
$$

$\hat{P}$ is the polarization vector, $E_{0}$ is the electric field amplitude, $\phi$ is the optical phase of the input plane wave, and $k$ is the absolute value of the free-space wave vector corresponding to $k=\omega / c$. Since the structure and the incident plane wave are assumed to extend to infinity along the grating grooves, the field components show no dependence on $z$, which will be omitted from here on.

The field components inside the vacuum channel determine the deflection and focusing forces acting on the electron beam. These fields have amplitudes $u(x, y)$ that obey the Helmholtz wave equation $\nabla^{2} u(x, y) / k^{2}+$ $u(x, y)=0$. For gratings these satisfy a pseudoperiodicity condition of the form $u\left(x+\lambda_{p}, y\right)=u(x, y) e^{-i \lambda_{p} k \sin \varphi}$ [10]. Let $k_{p}=2 \pi / \lambda_{p}$ be the grating $k$-vector magnitude. Then the field can be expressed as a discrete Fourier series having the form

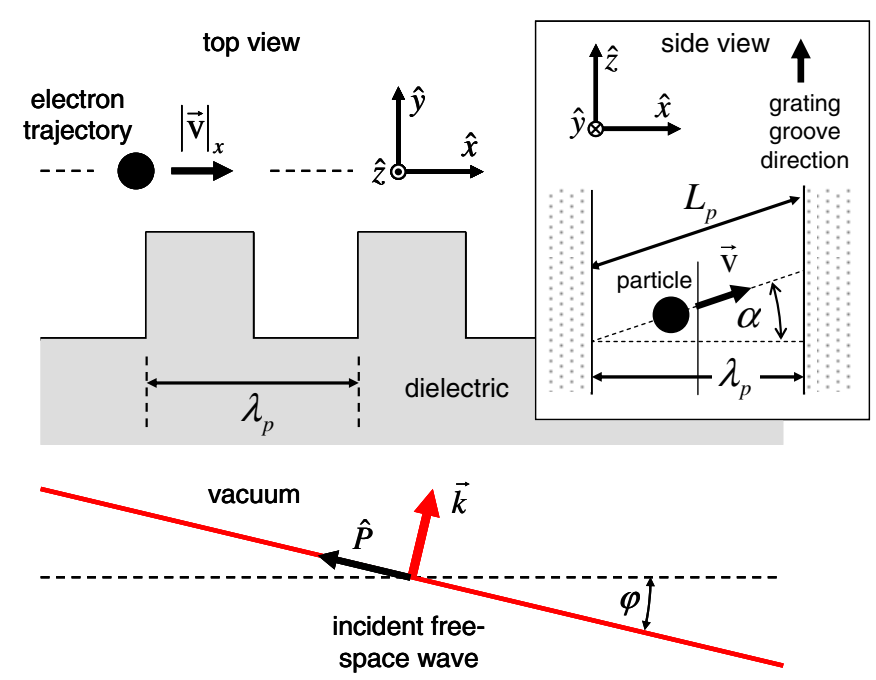

FIG. 3. (Color) Geometry of the incident TM-polarized plane wave on the grating structure and the electron beam trajectory. The side view inset shows the oblique orientation of the electron trajectory with respect to the grating grooves. 


$$
\begin{aligned}
& E_{x}(x, y, t)=\sum_{n=-\infty}^{+\infty} U_{n}(y) e^{i x\left(n k_{p}-k \sin \varphi\right)} e^{i k c t-i \phi} \\
& E_{y}(x, y, t)=\sum_{n=-\infty}^{+\infty} V_{n}(y) e^{i x\left(n k_{p}-k \sin \varphi\right)} e^{i k c t-i \phi} \\
& B_{z}(x, y, t)=\sum_{n=-\infty}^{+\infty} W_{n}(y) e^{i x\left(n k_{p}-k \sin \varphi\right)} e^{i k c t-i \phi}
\end{aligned}
$$

$U_{n}(y), V_{n}(y)$, and $W_{n}(y)$ describe the amplitudes of the grating diffraction orders and their dependence on the $y$ coordinate. These are decomposed into

$$
\begin{aligned}
U_{n}(y) & =u_{n,+} e^{+\Gamma_{n} y}+u_{n,-} e^{-\Gamma_{n} y} \\
V_{n}(y) & =v_{n,+} e^{+\Gamma_{n} y}+v_{n,-} e^{-\Gamma_{n} y} \\
W_{n}(y) & =w_{n,+} e^{+\Gamma_{n} y}+w_{n,-} e^{-\Gamma_{n} y} .
\end{aligned}
$$

The coefficients $\Gamma_{n}$ describe the mode of the $n$th grating diffraction order, and since the optical field $k$ vector is $k=$ $\left(k_{x}^{2}+k_{y}^{2}+k_{z}^{2}\right)^{1 / 2}$, these are

$$
\Gamma_{n}=\sqrt{\left(n k_{p}-k \sin \varphi\right)^{2}-k^{2}} .
$$

In the present notation a real $\Gamma_{n}$ corresponds to an evanescent mode. The field amplitudes are related to each other, and application of Maxwell's equations shows that inside the vacuum channel they are related to $W_{n}(y)$ by

$$
\begin{aligned}
& U_{n}(y)=\frac{c}{i k} d W_{n}(y) / d y \\
& V_{n}(y)=-\frac{n k_{p}-k \sin \varphi}{k / c} W_{n}(y) .
\end{aligned}
$$

In the present analysis it is assumed that the fields do not significantly alter the particle's trajectory over a grating period. Following Fig. 3 the particle's velocity is described by $\vec{v}(t)=\beta c(\hat{x} \cos \alpha+\hat{z} \sin \alpha)$ and a corresponding position $\vec{r}(t)=\vec{v} t$. The Lorentz force from the TM wave acting on the particle, expressed in the $(x, y, z)$ coordinates, is

$$
\begin{aligned}
\vec{F}[\vec{r}(t)] & =q \operatorname{Re}\{\vec{E}[\vec{r}(t)]+\vec{v} \times \vec{B}[\vec{r}(t)]\} \\
& =q \operatorname{Re}\left(\begin{array}{c}
E_{x}[\vec{r}(t)] \\
E_{y}[\vec{r}(t)]-\beta c B_{z}[\vec{r}(t)] \cos \alpha \\
0
\end{array}\right) .
\end{aligned}
$$

We seek the average force on the particle over an extended interaction distance. Let $s(t)=\beta c t$ be the distance traveled by the particle. The averaged force experienced by the free particle between $t=0$ and $t=T$ is

$$
\left\langle F_{j}\right\rangle=\frac{1}{s(T)} \int_{0}^{s(T)} F_{j}[\vec{r}(s)] d s .
$$

The average force components are therefore

$$
\begin{aligned}
\left\langle F_{x}\right\rangle_{\mathrm{TM}}= & q \operatorname{Re}\left(\frac{1}{s(T)} \int_{0}^{s(T)} \sum_{n=-\infty}^{+\infty} U_{n}(y) e^{i s \cos \alpha\left(k_{p} n-k \sin \varphi\right)}\right. \\
& \left.\times e^{i k s / \beta-i \phi} d s\right) \\
\left\langle F_{y}\right\rangle_{\mathrm{TM}}= & q \operatorname{Re}\left(\frac{1}{s(T)} \int_{0}^{s(T)} \sum_{n=-\infty}^{+\infty}\left[V_{n}(y)-c W(y) \beta \cos \alpha\right]\right. \\
& \left.\times e^{i s \cos \alpha\left(k_{p} n-k \sin \varphi\right)} e^{i k s / \beta-i \phi} d s\right) \\
\left\langle F_{z}\right\rangle_{\mathrm{TM}}= & 0 .
\end{aligned}
$$

The interaction is cumulative if the phase term of the exponents in Eq. (8) does not change with $s$, that is,

$$
n k_{p}-k \sin \varphi+k /(\beta \cos \alpha)=0 .
$$

Equation (9) represents the sought phase-synchronicity condition for a particle traveling with a velocity $\beta c$ in the structure shown in Fig. 2. It assumes that the structure is illuminated by a plane wave with $k$-vector magnitude $k$ and an angle of incidence $\varphi$. Inspection of Eq. (9) and the coefficient $\Gamma_{n}$ reveals that for $\beta<1$ and any grating tilt angle $\alpha$ phase synchronicity is only possible with the evanescent modes. This is in agreement with the Lawson-Woodward theorem, which states that free-space waves cannot sustain a linear long-range interaction with uniformly moving free particles [11]. Thus we encounter a situation similar to linear-interaction-force laser-driven accelerator structures, where a cumulative nonzero laserelectron interaction (either deflection or acceleration) can only occur in the presence of a material boundary.

Next, the analysis is extended to short laser pulses, which can be represented as a superposition of plane waves with different $k$-vector magnitudes. Define $\lambda$ as the center wavelength and $k_{0}=2 \pi / \lambda$ as the corresponding $k$-vector magnitude. Equation (9) establishes that each plane-wave component of the laser pulse with a specific $k$ must satisfy a certain angle of incidence $\varphi$. Assume that phase synchronicity for the center wavelength is satisfied at the angle of incidence $\varphi=0$. Then with Eq. (9) the phasesynchronicity condition at the center wavelength reads

$$
k_{0}=-n k_{p} \beta \cos \alpha .
$$

Again, $k_{p}=2 \pi / \lambda_{p}$ is the grating structure period. For a nonzero $k_{0}$, Eq. (10) can only be satisfied for $n \leq-1$, which corresponds to the evanescent modes described in Eq. (4). Assume that the angles of incidence of the other plane-wave components of different wavelength are small, such that $\sin \varphi \sim \varphi$. Defining $\Delta k=k-k_{0}$ and $\Delta \varphi=\varphi-$ $\varphi_{0}$, where $\varphi_{0}=0$ is the angle of incidence of the center wavelength, Eq. (9) can be rewritten as

$$
\frac{1}{\beta \cos \alpha}=k \frac{\Delta \varphi}{\Delta k} \equiv \tan \psi
$$


Equation (11) represents a pulse-front tilt condition for an electromagnetic wave where the pulse-front tilt angle is $\psi$ [12], which guarantees synchronicity of the laser pulse envelope with the particle. Equations (10) and (11) establish the carrier phase and the envelope synchronicity conditions with the particle traveling down the vacuum channel at a velocity $\beta c$.

The force components $\left\langle F_{x}\right\rangle,\left\langle F_{y}\right\rangle$, and $\left\langle F_{z}\right\rangle$ in Eq. (8) are expressed in a coordinate system $(x, y, z)$ that is aligned with the structure coordinates shown in Fig. 2. However, we are interested in these forces as observed in the particle's coordinate system $\left(x^{\prime}, y^{\prime}, z^{\prime}\right)$, which is rotated by an angle $\alpha$ about the $y$ axis:

$$
\begin{aligned}
& \left\langle F_{x^{\prime}}\right\rangle=+\left\langle F_{x}\right\rangle \cos \alpha+\left\langle F_{z}\right\rangle \sin \alpha \\
& \left\langle F_{z^{\prime}}\right\rangle=-\left\langle F_{x}\right\rangle \sin \alpha+\left\langle F_{z}\right\rangle \cos \alpha \quad\left\langle F_{y^{\prime}}\right\rangle=\left\langle F_{y}\right\rangle .
\end{aligned}
$$

$\left\langle F_{x^{\prime}}\right\rangle$ is the average acceleration force experienced by the particle. $\left\langle F_{y^{\prime}}\right\rangle$ and $\left\langle F_{z^{\prime}}\right\rangle$ are the average horizontal and vertical deflection forces. Note that Eq. (9) can be satisfied for one mode at a time, and in general, the diffraction modes are weaker with increasing diffraction order number. Therefore the sum in Eq. (8) reduces to the one particular value of $n$ for which phase synchronicity is satisfied, and typically the $n= \pm 1$ mode is the strongest evanescent mode. This will be discussed more extensively in Sec. III with a numerical example. For the TM-polarized laser beam the first-order diffraction modes $n=-1$ yield a set of force components of the form

$$
\begin{aligned}
& \left\langle F_{x^{\prime}}\right\rangle_{\mathrm{TM}}=\frac{q c}{k} \cos \alpha \operatorname{Re}\left[e^{-i \phi} d W_{-1}(y) / d y\right] \\
& \left\langle F_{y^{\prime}}\right\rangle_{\mathrm{TM}}=q c\left(\frac{1}{\beta \cos \alpha}-\beta \cos \alpha\right) \operatorname{Re}\left[e^{-i \phi} W_{-1}(y)\right] \\
& \left\langle F_{z^{\prime}}\right\rangle_{\mathrm{TM}}=-\frac{q c}{k} \sin \alpha \operatorname{Re}\left[e^{-i \phi} d W_{-1}(y) / d y\right] .
\end{aligned}
$$

Section IV explores the effect of the grating tilt angle and the depth of the grating groove on the amplitude of the grating diffraction order $W_{n}(y)$ and on the deflection force component.

\section{SYMMETRIC LASER PUMPING OF THE STRUCTURE}

The evanescent field pattern described in Sec. II possesses the desired synchronicity conditions but is nonuniform and asymmetric. Furthermore, the deflection force is not aligned with the structure coordinates. A practical beam manipulation element is only useful if it generates a deflection force that possesses a high degree of uniformity, symmetry along the vacuum channel, and furthermore is aligned to the beam coordinates. Excitation of symmetric modes in the vacuum channel of a resonant dielectric periodic structure is one possibility that has been explored for other laser-driven accelerator structures [13]. However, as stated in the Introduction, we are interested in the application of ultrashort, few-cycle laser pulses and therefore cannot resort to resonant-field architectures. Instead, we can illuminate the transparent grating structure from opposite sides to generate a symmetric field pattern and furthermore accomplish the cancellation of undesired force components.

First, consider the addition of two TM-polarized laser beams of equal amplitude whose electric field components are in phase at the center of the vacuum channel. This results in a laser field amplitude that modifies the expression of $W_{n}(y)$ in Eq. (3) to a hyperbolic function:

$$
W_{n}(y)=w_{n} \sinh \left(\Gamma_{n} y\right) ; \quad w_{n}=2\left(w_{n,+}-w_{n,-}\right) .
$$

For this superposition of fields the amplitude components in Eq. (13) become

$$
\begin{aligned}
& \left\langle F_{x^{\prime}}\right\rangle_{\mathrm{TM}}=\frac{q c}{k} \cos \alpha \operatorname{Re}\left[e^{-i \phi} w_{-1} \Gamma_{-1} \cosh \left(\Gamma_{-1} y\right)\right]
\end{aligned}
$$

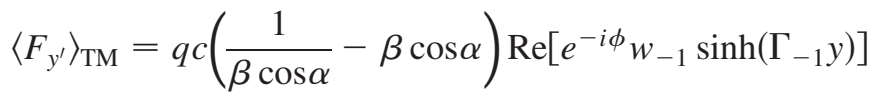

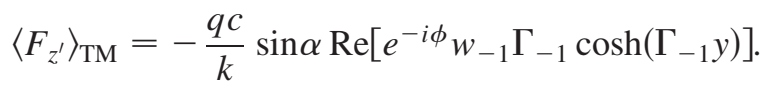

Near the center of the vacuum channel the deflection component $\left\langle F_{y^{\prime}}\right\rangle$ shows a dependence that is nearly linear with the $y$ coordinate due to the $\sinh \left(\Gamma_{n} y\right)$ function while the other components remain nearly uniform. This external laser beam configuration results in a force pattern that provides a focus along the $y$ axis while generating a uniform synchronous force component in the other two dimensions. This is a favorable configuration since it allows for extended transport of a beam that is tightly focused in the $y$ direction while providing a uniform deflection parallel to the vacuum channel.

Consider the opposite case, where the laser beam electric field components are out of phase by $\pi$ with respect to each other. The amplitude function $W_{n}(y)$ in Eq. (14) changes parity to an odd function $W_{n}(y)=w_{n} \cosh \left(\Gamma_{n} y\right)$; $w_{n}=2\left(w_{n,+}+w_{n,-}\right)$ and therefore the force components that result from phase synchronicity with the first diffraction order modify to

$$
\begin{aligned}
& \left\langle F_{x^{\prime}}\right\rangle_{\mathrm{TM}}=\frac{q c}{k} \cos \alpha \operatorname{Re}\left[e^{-i \phi} w_{-1} \Gamma_{-1} \sinh \left(\Gamma_{-1} y\right)\right] \\
& \left\langle F_{y^{\prime}}\right\rangle_{\mathrm{TM}}=q c\left(\frac{1}{\beta \cos \alpha}-\beta \cos \alpha\right) \operatorname{Re}\left[e^{-i \phi} w_{-1} \cosh \left(\Gamma_{-1} y\right)\right] \\
& \left\langle F_{z^{\prime}}\right\rangle_{\mathrm{TM}}=-\frac{q c}{k} \sin \alpha \operatorname{Re}\left[e^{-i \phi} w_{-1} \Gamma_{-1} \sinh \left(\Gamma_{-1} y\right)\right]
\end{aligned}
$$

The deflection force that is oriented into the walls of the structure, $\left\langle F_{y^{\prime}}\right\rangle$, remains nearly uniform while the other deflection force and the acceleration force component scale as $\sinh \left(\Gamma_{n} y\right)$. This field pattern is clearly undesirable since it skews the electron beam. 


\section{STRUCTURE MATERIAL AND GEOMETRY CONSIDERATIONS}

Inspection of the force components of Eqs. (15) and (16) shows an explicit dependence of these on the grating tilt angle $\alpha$ and on the strength of the grating diffraction mode $W_{-1}(y)$. These diffraction modes depend on the shape of the grating grooves and on the index of refraction of the grating material. The effect of these parameters is discussed in this section by means of an example. Figure 4(a) shows a schematic of the grating diffraction modes $W_{n}(y)$ inside the vacuum channel.

Assume that a pair of laser beams with the polarization and phase conditions of Eq. (15) with a field amplitude $\left|E_{\text {laser }}\right|=1$ are incident on a quartz (index of refraction = 1.58 for $\lambda=800 \mathrm{~nm}$ ) based grating structure as shown in Fig. 2. Furthermore, assume the grating structure having a channel width $w=0.4 \lambda$ and a groove depth of $0.2 \lambda$. The grating period is established by the phase-synchronicity condition of Eq. (9), which depends on the grating tilt angle $\alpha$. In this example it will be assumed that the electron beam is relativistic; $\gamma \rightarrow \infty$. We employ a multilayer grating field decomposition method to determine the magnitude and relative phase of the diffraction orders $W_{n}$ [14].

Figure 4(b) shows the dependence of the diffraction modes at the center of the vacuum channel $W_{n}(y=0)$ on the depth $g$ of the grating grooves for a grating with a period $\lambda_{p}=\lambda / \cos \alpha$ with $\alpha=50^{\circ}$. Interference effects from reflections between grating layers are appreciable on the zeroth diffraction mode, which in the example considered here is the only nonevanescent mode. When the grating groove depth $g$ is zero, the first and higher order diffraction modes are zero. The magnitude of $W_{-1}$ peaks at $g=0.2 \lambda$ and slightly decreases for deeper grating grooves. The other modes remain negligibly small.

Next, Fig. 5(a) shows the amplitude coefficients of the diffraction orders at the center of the vacuum channel $W_{n}(y=0)$ for $g=0.2 \lambda$ as a function of $\alpha$. The diffraction orders become drastically weaker with increasing diffraction order number. In this particular example, $W_{0}$ is the only propagating mode while the remaining modes are evanescent. Since the grating period is reduced with increasing grating tilt angle $\alpha$, the evanescent modes become weaker. Figure 5(b) shows the magnitudes of the deflection forces corresponding to each diffraction mode. As expected from Fig. 4(a) the first grating diffraction mode accounts for most of the total force.

Dielectric materials can support a maximum fluence of $\sim 1.5 \mathrm{~J} / \mathrm{cm}^{2}$ from ultrashort laser pulses [15], which for $10 \mathrm{fsec}$ translates to a maximum local electric field of $\sim 27 \mathrm{GV} / \mathrm{m}$. Application of Eqs. (3)-(5) and (14) allows for an estimate of the ratio between the field at the walls of the structure and the free-space field of the input laser beam, which for the chosen parameters is $\sim 2.8$. Therefore the maximum applicable laser field is $E_{\text {in }} \sim$ $10 \mathrm{GV} / \mathrm{m}$. As shown in Fig. 5(b) the maximum deflection force is $\left\langle F_{z^{\prime}}\right\rangle \sim 0.1 q E_{\text {in }}$ for the selected grating geometry, which hence corresponds to a maximum average deflection field of $\sim 1 \mathrm{GV} / \mathrm{m}$. A magnetic field of $\sim 3 \mathrm{~T}$ would be required to match this average deflection force generated from the laser beam inside the grating structure.

Some conclusions on the fabrication tolerances can be drawn from Figs. 4 and 5. Figure 4(b) shows that the magnitude of the deflection is a slowly varying function of the groove depth, and near the optimum parameters the groove depth can vary by $0.1 \lambda$ without a significant change
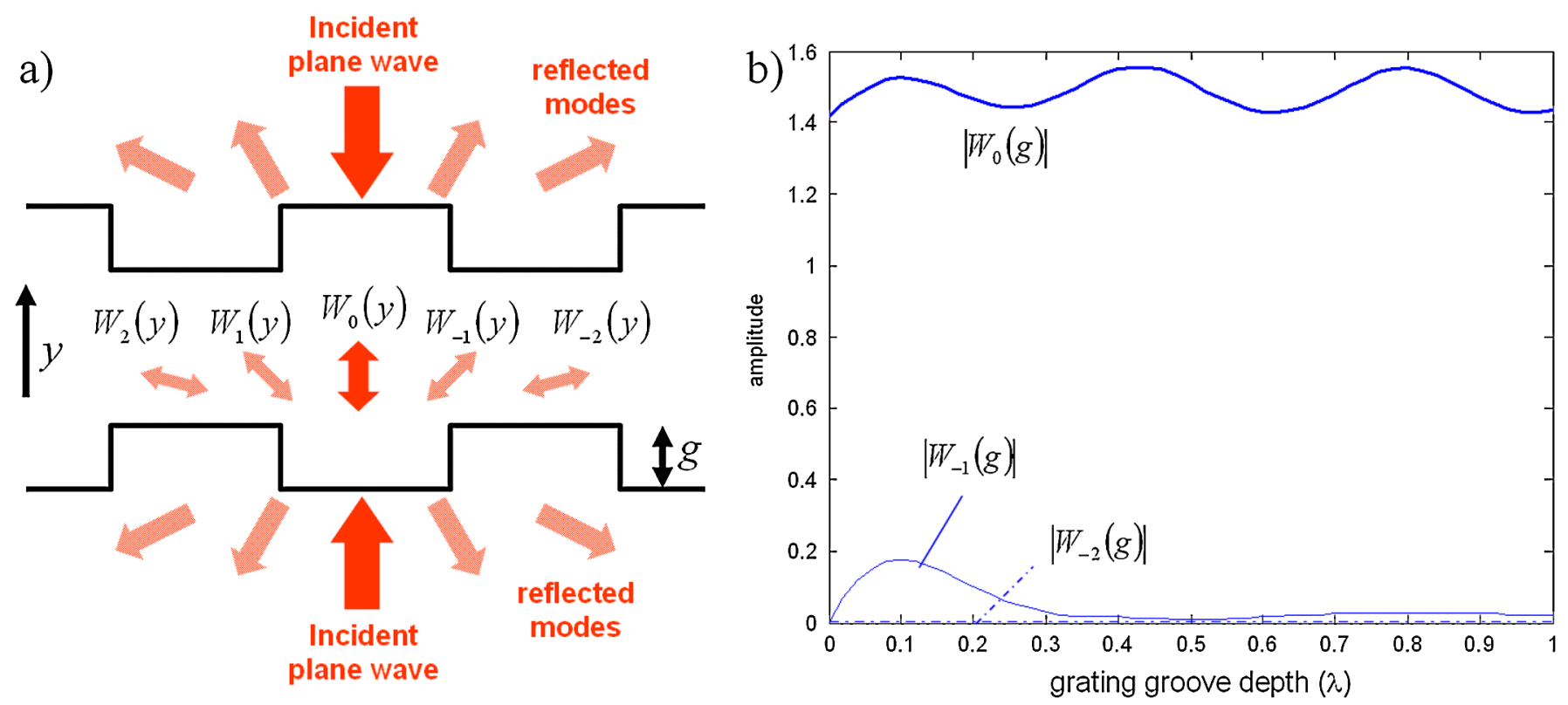

FIG. 4. (Color) (a) Top view of the grating vacuum channel and the orders $W_{n}$. (b) Magnitude of the diffraction modes as a function of the grating groove depth $g$. 

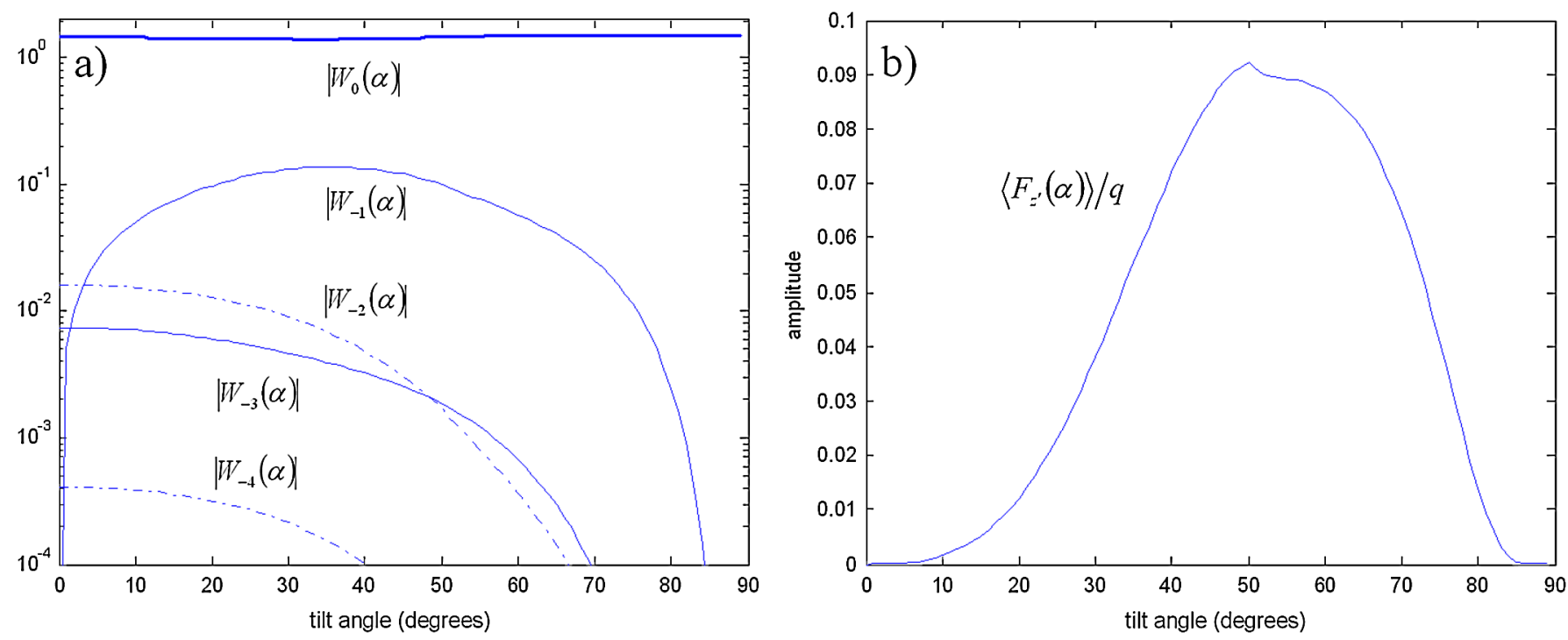

FIG. 5. (Color) (a) Magnitude of the grating diffraction modes as a function of the grating tile angle satisfying the synchronicity condition for the first diffraction mode. The grating groove depth $g$ is $g=0.2 \lambda$. (b) Magnitude of the corresponding deflection force in the $z^{\prime}$ direction.

in the value of $W_{-1}$. Control of the etch depth on quartz structures to within 1\% has been reported [16]. Another important fabrication aspect is the tolerance of the grating period. Modification of Eq. (9) reveals that for the grating order $n=-1$ the accumulated phase mismatch $\Delta \phi$ over a grating length $L$ reads $\Delta L / L=(\Delta \phi / 2 \pi)\left(\lambda_{p} / L \beta \cos \alpha\right)$. If we establish a tolerance condition of $\Delta \phi$ equal to $1^{\circ}$ for a grating of period $\lambda_{p}=800 \mathrm{~nm}$ over $L=1 \mathrm{~cm}$, we obtain a groove position tolerance of $\Delta L \sim 2 \mathrm{~nm}$. DeepUV lithography fabrication techniques have shown linewidth uniformity values of $0.1 \mathrm{~nm}$ over $1 \mathrm{~cm}$ distances [17]. The tight tolerance of the length and the thermal expansion coefficient of $\sim 5 \times 10^{-7} /{ }^{\circ} \mathrm{C}$ for quartz establish a limited operating temperature range for the $\mathrm{cm}$-long grating of $\sim \frac{1}{2}{ }^{\circ} \mathrm{C}$ but also allows for postfabrication temperature-controlled tuning of the structure, in similar fashion as is carried out with conventional metal structure accelerators.

\section{APPLICATIONS}

\section{A. Swept beam steering and focusing devices}

Beam steering devices are found in applications such as kickers for high energy beams [18-20], electron sweepers for A-D converters [21], and streak cameras [22,23]. Their development toward increased sweep speed remains an active field of research. The state-of-the-art deflectors are millimeter-scale electronic elements that are based on circuit- and rf-based traveling wave deflection concepts [24-26]. The proposed laser-driven deflection structure represents the extension of these rf-based deflection concepts to optical wavelengths to reach time resolution values in the sub-fsec scale.
Figure 6(a) shows the profile of the electron beam inside the deflection device, having dimensions $\sigma_{0, y^{\prime}}$ and $\sigma_{0, z^{\prime}}$, where $\sigma_{0, z^{\prime}} \gg \sigma_{0, y^{\prime}}$. The laser beams powering the structure are set to be in phase, which as described in Sec. III generates a deflection force parallel to the vacuum channel.

Application of focusing can enhance the angular resolution along the deflection coordinate. Instead of using a conventional focusing element we propose employing the same type of double-grating structure for this purpose, as is illustrated in Fig. 6(b). Applying a laser field spatial envelope of the form $A\left(z^{\prime}\right) \propto z^{\prime}$ on the structure generates a position dependent deflection angle of the form $\theta\left(z^{\prime}\right)=$ $\theta_{0}+z^{\prime} / f$, where $\theta_{0}$ is the original beam direction upstream of the focusing element and $f$ is the effective focal length.

Assume a grating structure with the optimized parameters of Sec. IV, where the deflection force $\left\langle F_{z^{\prime}}\right\rangle$ scales with the external laser amplitude $A\left(z^{\prime}\right)$ as $\left\langle F_{z^{\prime}}\right\rangle \sim 0.1 q A\left(z^{\prime}\right)$. Assuming the acceleration from the deflection force results in a lateral velocity much smaller than $c$, it can be shown that the focal length $f$ from a field with profile variation $d A / d z^{\prime}$ over a structure length $L_{F}$ is

$$
\frac{1}{f}=\left|\frac{d \theta}{d z^{\prime}}\right| \sim 0.1 \frac{q L_{F}}{\gamma m \beta^{2} c^{2}}\left(d A / d z^{\prime}\right) .
$$

A pair of $\mathrm{TEM}_{01}$ beams with peak field amplitude $E_{0}=$ $9 \mathrm{GV} / \mathrm{m}$ and vertical spot size of $\sim 1 \mathrm{~mm}$ possesses an amplitude variation $d A / d z^{\prime} \sim 10^{13} \mathrm{~V} / \mathrm{m}^{2}$ near the center of the beam. For a focusing element length $L_{F}=100 \mu \mathrm{m}$ and a $10 \mathrm{MeV}$ electron beam, Eq. (16) predicts a focal length of $\sim 10 \mathrm{~cm}$.

Define the angular resolution as the ratio of the transverse sweep range $z_{f}^{\prime}$ versus the beam spot size at the 
a)

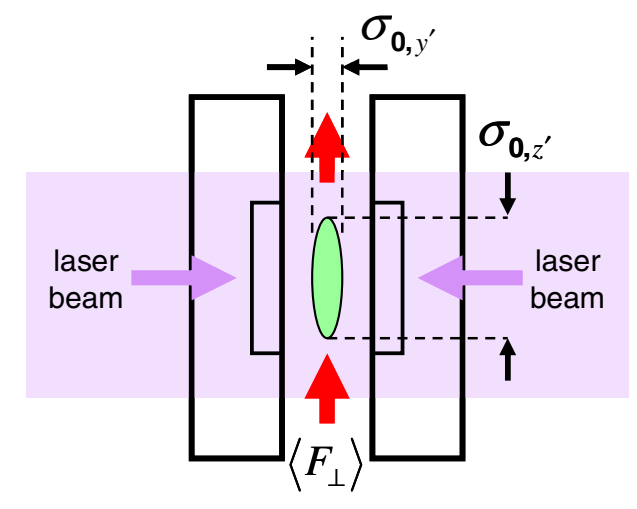

b)

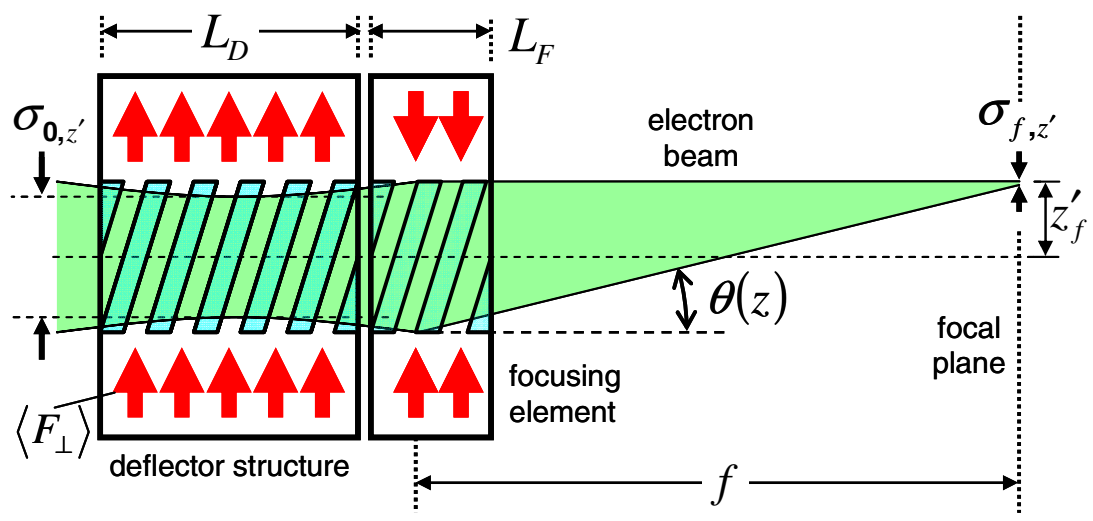

FIG. 6. (Color) (a) Cross-sectional view of the deflector structure and the electron beam. (b) Transverse view of the deflector structure, the focusing structure, and the electron beam. The solid arrows indicate the expected force generated by the laser beam pattern on the electron beam.

observation plane, that is, $R^{\prime}=z_{f} / \sigma_{f, z^{\prime}}$. Similar to a Gaussian laser beam the electron beam spot size evolution is parametrized by a beam waist spot size $\sigma_{f, z^{\prime}}$ in the focal plane and a depth of focus $\beta_{f, z^{\prime}}$. The spot sizes $\sigma_{0, z^{\prime}}$ and $\sigma_{f, z^{\prime}}$ are related to the focal length $f$ and the depth of focus $\beta_{f, z^{\prime}}$ by an equation of the form [27]

$$
\sigma_{0, z^{\prime}}=\sigma_{f, z^{\prime}}\left(1+f^{2} / \beta_{f, z^{\prime}}{ }^{2}\right)^{1 / 2}
$$

For a deflection force $\left\langle F_{z^{\prime}}\right\rangle$, a deflector structure length $L_{D}$, and a focal length $f$, the resulting deflection is $z_{f}^{\prime} \sim$ $f L_{D}\left\langle F_{z^{\prime}}\right\rangle / \gamma m c^{2}$ and the resolution becomes

$$
R=\frac{f L_{D}\left\langle F_{z^{\prime}}\right\rangle}{\sigma_{f, z^{\prime}} \gamma m c^{2}} .
$$

Optimization of $R$ suggests minimizing the focal plane spot size $\sigma_{f, z^{\prime}}$. As shown in Eq. (18) for a given $\sigma_{0, z^{\prime}}$, $\sigma_{f, z^{\prime}}$ is a function of the focal length $f$ and the depth of focus of the electron beam, $\beta_{f, z^{\prime}}=\sigma_{f, z^{\prime}}^{2} / 4 \varepsilon_{z^{\prime}}$, which depends on the transverse geometric emittance of the beam $\varepsilon_{z^{\prime}}$. Ideal electron sources for this application are laserdriven field emitters capable of ultralow emittance values followed by a dielectric-structure laser accelerator. Such devices are expected to support geometric electron beam emittance values of $\varepsilon_{\perp} \sim 10^{-9} / \gamma \mathrm{m}[28,29]$.

To obtain an order-of-magnitude estimate of the resolution possible from the proposed device, consider a grating structure whose total length satisfies $L_{D}+L_{F}<2 \beta_{0, y^{\prime}}$, where $\beta_{0, y^{\prime}}=\sigma_{0, y^{\prime}}^{2} / 4 \varepsilon_{y^{\prime}}$. Since the vacuum channel has a submicron width the electron spot size should be on the order of $\sim \frac{1}{10} \mu \mathrm{m}$. Consider an electron beam of $10 \mathrm{MeV}$ energy, and having an emittance $\varepsilon_{z^{\prime}} \sim \varepsilon_{y^{\prime}} \sim 10^{-9} / \gamma \mathrm{m}$ and a transverse electron spot size that allows it to traverse the grating structure, for example $\sigma_{0, y^{\prime}} \sim \frac{1}{10} \mu \mathrm{m}$. This constrains the total structure length $L_{D}+L_{F}$ to $\sim 250 \mu \mathrm{m}$. The optimization consists in selecting the lengths $L_{D}$ and $L_{F}$ such that $R$ is maximized, which in this example yields $L_{D} \sim 9 L_{F}$, a focal length of $13.4 \mathrm{~cm}$, and a resolution $R \sim 10^{3}$. Figure 7 shows the evolution of the electron beam $y$ and $z$ envelopes for the given example downstream of the structure. At the focal plane the spot size in the sweep direction is $2 \mu \mathrm{m}$ while its other dimension is $\sim 100$ times larger. Since the driving waveform is sinusoidal in time only $\sim 10 \%$ of the cycle corresponds to a sweep that is approximately linear.

The $2 \mu \mathrm{m}$ spot size at the focal plane corresponds to the smallest CCD pixel size values for commercially available image detectors. The temporal resolution for one such pixel and laser wavelength of $\lambda=1 \mu \mathrm{m}$ corresponds to

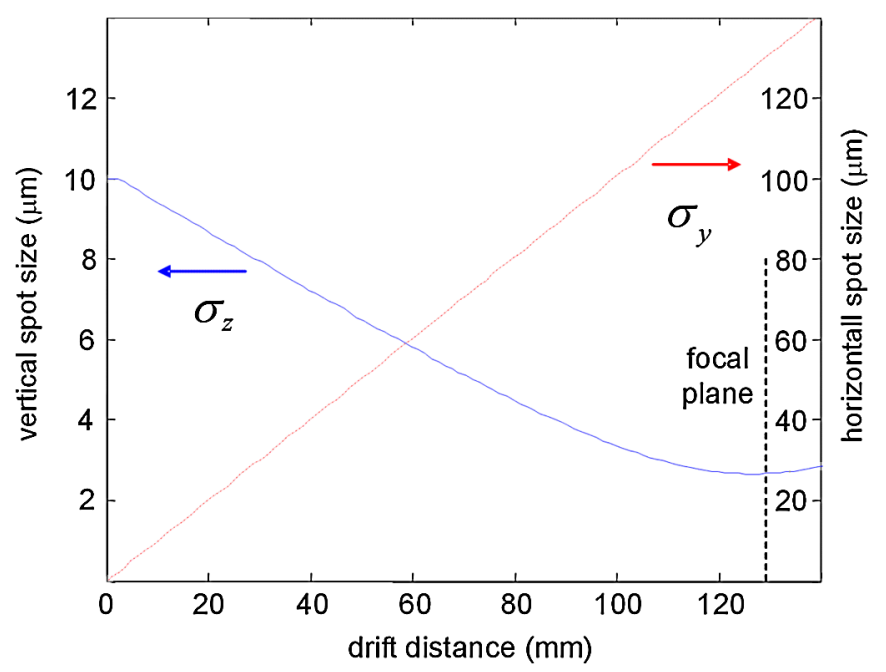

FIG. 7. (Color) Transverse beam profile as a function of distance behind the structure shown in Fig. 5. Without further focusing elements the beam comes to a line focus about $13 \mathrm{~cm}$ downstream. 

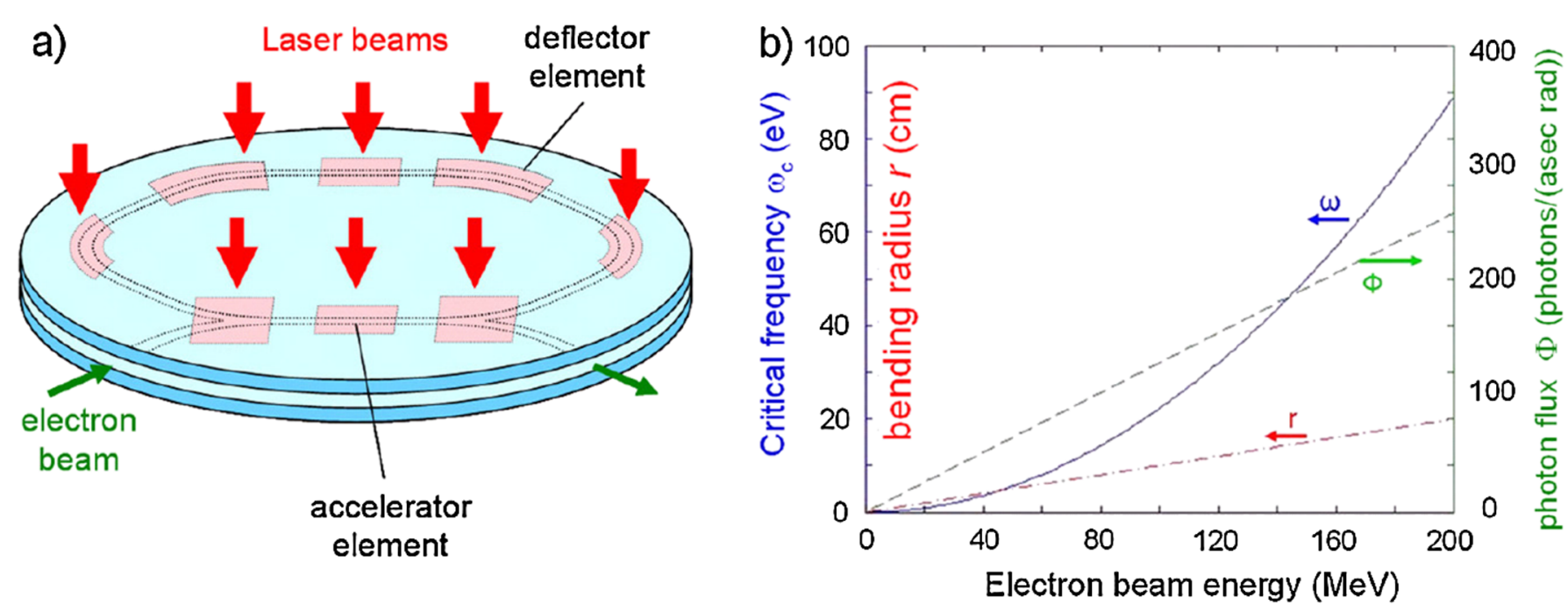

FIG. 8. (Color) (a) Schematic of an electron ring based on dielectric grating manipulation elements. (b) Synchrotron parameters as a function beam energy.

a temporal resolution $\Delta t=\lambda / 2 \pi c R$, or $\Delta t \sim 5 \times$ $10^{-19} \mathrm{sec}$. Imaging of $\mathrm{MeV}$-energy electron beams can be accomplished with scintillator materials such as Ce:YAG, which shows a fluorescence lifetime of $\sim 100 \mathrm{nsec}$. The readout speed is limited by this lifetime, and for electron bunch repetition rates above $10 \mathrm{MHz}$ the streak camera displays an average temporal structure of the electron beam within the laser optical cycle.

\section{B. Bending elements}

The maximum deflection field was found to be $\left\langle F_{\perp} / q\right\rangle \sim 1 \mathrm{GV} / \mathrm{m}$, which leads to a beam bending radius of $r=\gamma m \beta^{2} c^{2} /\left\langle F_{\perp}\right\rangle$. For few-MeV beam energies it can be on the order of $1 \mathrm{~cm}$, allowing for the possibility of fitting an electron ring into a few-cm diameter device. Besides the deflector units, an electron ring requires input and exit beam kickers, accelerator, and focusing sections. As shown in Fig. 8(a) all these binary grating elements are envisioned to be fabricated onto a pair of quartz wafers and be powered by the corresponding laser beam modes. Figure 8(b) shows the bending radius, synchrotron critical frequency $\omega_{c}$ and photon flux (within $0.1 \%$ of $\omega_{c}$ ) for a $10 \mathrm{fC}, 100$ attosecond electron bunch as a function of beam energy. At $20 \mathrm{MeV}$ the electron bunch would generate a GHz-repetition visible light pulse train with a radiation energy loss of $\sim 1 \mathrm{eV}$ per turn. At $200 \mathrm{MeV}$, the required bending radius is $\sim 20 \mathrm{~cm}$, the peak radiation wavelength is $14 \mathrm{~nm}$, and the synchrotron radiation energy loss is hundreds of eV per turn. Such a device could function as a compact and high-repetition rate collimated extreme ultraviolet source.

\section{OUTLOOK}

A first analysis of the transparent double grating as a deflection and focusing element was presented. Existing nanofabrication and laser technologies render the proposed structure a promising future beam manipulation device. Demonstration experiments of these deflection and focusing properties with $\mathrm{MeV}$-energy electron beams form a crucial next step. Finally, a series of more refined analysis and simulation efforts will be required to study the dynamics of a particle beam that is propagated through a more complex grating based device such as an entire grating based laser-accelerator unit, undulator, or electron ring. These will ultimately pinpoint the capabilities and limitations of the proposed beam manipulation device and will determine their feasibility as practical devices.

\section{ACKNOWLEDGMENTS}

This work is supported by the PULSE center at SLAC and by Northrop Grumman Space Technology. The authors would like to thank D. Sutter for valuable suggestions regarding grating accelerator structures and for pointing us to very important work on open grating accelerator structures. Also, we very much appreciate M. Tigner from Cornell University for sending us his copy of M. A. Pickup's thesis on metallic grating based linear accelerators. We also acknowledge funding from MPG, EU, and Munich Center for Advanced Photonics.

\section{APPENDIX}

A real structure and laser beam do not have infinite spatial extent. However, if the field amplitude of the laser beam is varying slowly in the $z$ direction it can be approximated by a function of the form $u(x, y, z) \sim a(z) b(x, y)$. The incoming laser beam possesses a beam profile described by a slowly varying envelope function $a(z)$. The function $b(x, y)$, on the other hand, shows a rapid spatial variation caused by the diffraction from the grating grooves which have features with a size of $\lambda$. Hence, the resulting 
field variations include components of the form $e^{i k_{x} x+i k_{y} y}$, where $k_{x}$ and $k_{y}$ scale with the wavelength of the laser $k=$ $2 \pi / \lambda$. The amplitude $u(x, y, z)$ satisfies the Helmholtz equation $\left(\nabla^{2}+k^{2}\right) u(x, y, z)=0$. Thus, to neglect the dependence on $z$ it is required that the derivative of $a(z)$ with $z$ be small compared to the derivative of $b(x, y)$ with respect to $(x, y)$. This yields a condition for the minimum laser profile $w_{z} \gg \lambda / 2 \pi$. A laser focus as small as $w_{z} \sim$ $50 \lambda$ is readily attainable with standard focusing elements and lies well within the mentioned condition for the twodimensional field approximation. The Rayleigh range of such a focus would correspond to $Z_{R} \sim 800 \lambda$ and, assuming that the beam waist is at the center of the vacuum channel, the radius of phase front curvature at a distance $\lambda$ away would correspond to a radius of $\sim 6 \times 10^{5} \lambda$. This indicates that there is no significant phase variation in the $z$ direction and that the two-dimensional diffraction analysis in the $(x, y)$ coordinates is applicable for the described laser beam.

[1] T. Plettner and R. L. Byer, Phys. Rev. ST Accel. Beams 11, 030704 (2008).

[2] C. M.S. Sears et al., Phys. Rev. ST Accel. Beams 11, 061301 (2008).

[3] T. Plettner, P. P. Lu, and R. L. Byer, Phys. Rev. ST Accel. Beams 9, 111301 (2006).

[4] S. Astilean, P. Lalanne, P. Chavel, E. Cambril, and H. Launois, Opt. Lett. 23, 552 (1998).

[5] J. N. Mait, A. Scherer, O. Dial, D. W. Prather, and X. Gao, Opt. Lett. 25, 381 (2000).

[6] S. Wang, C. Zhou, Y. Zhang, and H. Ru, Appl. Opt. 45, 2567 (2006).

[7] R. B. Palmer, Report No. SLAC-PUB-4161, 1986.

[8] M. A. Pickup, Ph.D. thesis, Cornell University, 1987.

[9] Y. Y. Lin and Y. C. Huang, Phys. Rev. ST Accel. Beams 10, 030701 (2007).

[10] M. Nevière and E. Popov, Light Propagation in Periodic Media (Marcel Decker, Basel, Switzerland, 2003), pp. 21-22.

[11] E. Esarey, P. Sprangle, and J. Krall, Phys. Rev. E 52, 5443 (1995).

[12] J. Hebling, Opt. Quantum Electron. 28, 1759 (1996).

[13] J. Rosenzweig, A. Murokh, and C. Pellegrini, Phys. Rev. Lett. 74, 2467 (1995).

[14] D. M. Pai and K. A. Awada, J. Opt. Soc. Am. A 8, 755 (1991).

[15] M. Lenzner et al., Phys. Rev. Lett. 80, 4076 (1998).

[16] A. Kowalik, K. Gora, Z. Jaroszewicz, and A. Kołodziejczyk, Microelectron. Eng. 77, 347 (2005).

[17] S. Selvaraja, W. Bogaerts, D. Van Thourhout, and R. Baets, in Proceedings of the 14th European Conference on Integrated Optics (ECIO), Netherlands, 2008, p. 442.

[18] D. Pooley, J. Phys. E 4, 909 (1971).

[19] S.S. Kurennoy, J.F. Power, and D. L. Schrage, in Proceedings of the 18th Particle Accelerator Conference, New York, 1999 (IEEE, New York, 1999), p. 1399.

[20] M. Clarke-Gayther, in Proceedings of the European Particle Accelerator Conference, Vienna, 2000 (EPS, Geneva, 2000), p. 2358.

[21] R. F. W. Pease, K. Ioakeimidi, R. Aldana, and R. Leheny, IEEE Trans. Microwave Theory Tech. 53, 336 (2005).

[22] V. Guidi and A. V. Novokhatsky, Meas. Sci. Technol. 6, 1555 (1995).

[23] G. Naylor and K. Scheidt, Meas. Sci. Technol. 12, 1858 (2001).

[24] R. Aldana and F. Pease, Microelectron. Eng. 84, 806 (2007).

[25] K. Harkay et al., in Proceedings of the 21st Particle Accelerator Conference, Knoxville, 2005 (IEEE, Piscataway, NJ, 2005), p. 668.

[26] P. Emma and Z. Huang, Nucl. Instrum. Methods Phys. Res., Sect. A 528, 458 (2004).

[27] H. Wiedemann, Particle Accelerator Physics I (SpringerVerlag, Berlin, 1999), 2nd ed.

[28] B. Cowan, Phys. Rev. ST Accel. Beams 11, 011301 (2008).

[29] P. Hommelhoff, C. Kealhofer, and M. A. Kasevich, Phys. Rev. Lett. 97, 247402 (2006). 